Groups Geom. Dyn. 4 (2010), 657-679

DOI $10.4171 / \mathrm{GGD} / 99$
Groups, Geometry, and Dynamics

(C) European Mathematical Society

\title{
Subgroups of free metabelian groups
}

\author{
Gilbert Baumslag, Charles F. Miller III and Gretchen Ostheimer
}

\begin{abstract}
In 1954 A. G. Howson proved that the intersection of two finitely generated subgroups of a free group is again finitely generated. Now the free metabelian subgroups of a free metabelian group of finite rank $n$ are quite restricted. Indeed they are again of finite rank at most $n$. This suggests that there may be an analog of Howson's theorem for free metabelian groups. This turns out not to be the case. The object of this paper is to explore such intersections in free metabelian groups and, more generally, in the wreath product of two free abelian groups. In such a wreath product we show, for instance, that there are algorithms to decide whether or not the intersection of two finitely subgroups is finitely generated or trivial. This leaves open the existence of algorithms to decide the same questions for finitely generated subgroups of finitely generated metabelian groups as a whole.
\end{abstract}

Mathematics Subject Classification (2010). 20E10, 20E22, 20R10.

Keywords. Free metabelian, intersections, wreath products, algorithms.

\section{Introduction}

1.1. Finitely generated metabelian groups. In his ground breaking paper [9] in 1954 , P. Hall observed that the commutator subgroup $[G, G]$ of a finitely generated metabelian group $G$ can be viewed as a finitely generated module over the integral group ring of the factor derived group $G /[G, G]$. Thus the structure of finitely generated metabelian groups is in large measure determined by the structure of finitely generated modules over polynomial rings in finitely many variables. This enabled Hall to prove a number of beautiful theorems about finitely generated metabelian groups. In particular he showed that they satisfy $\max -n$, the maximal condition for normal subgroups, and hence that there are only a countable number of isomorphism classes of finitely generated metabelian groups. Another consequence of this maximal condition is that the additive group of rational numbers is not a subgroup of a finitely generated metabelian group, which places a restriction on the abelian subgroups of these groups. However, as Hall pointed out in [10], the nature of the abelian subgroups remains difficult to determine. 
Hall's work gave rise to a number of positive algorithmic results about finitely generated metabelian groups (see for instance the monograph [15]). In particular, Romanovskii [21] in 1980 proved that there is an algorithm to decide whether or not an element in a finitely generated metabelian group lies in a given finitely generated subgroup. Thus the intersection of two finitely generated subgroups is a recursive set, i.e., there is an algorithm to decide if an element is or is not in the intersection of two finitely generated subgroups. However many problems remain open. Although the word and conjugacy problems have been shown to have positive solutions, the isomorphism problem remains seemingly out of reach at this time. One positive result in this area is the proof by Groves and Miller [8] that there is an algorithm which determines whether or not a finitely generated metabelian group is free metabelian.

It seems that free metabelian groups are more tractable than finitely generated metabelian groups as a whole. For instance it is easy enough to prove that the free metabelian subgroups of a free metabelian group of finite rank $n$ have rank at most $n$. Indeed a subgroup of a free metabelian group is free if and only if it is generated by a set of elements which are independent modulo the derived group [1]. Moreover, the non-cyclic abelian subgroups of a free metabelian group are contained in the derived group and are therefore free abelian. In fact Wilhelm Magnus [16] has proved that every free metabelian group can be embedded in the wreath product of two free abelian groups and it is easy to prove that the abelian subgroups of such wreath products are free abelian. Many results about finitely generated metabelian groups make use of wreath products and this theorem of Magnus, for example the embedding theorem of Baumslag [2] and Remeslennikov [20]. But these remarks belie the complexity of even this restricted class of metabelian groups. For instance there are continuously many subgroups of the free metabelian group of rank two [6].

It is easy to characterize the finitely generated metabelian groups in which the intersection of finitely generated subgroups are again finitely generated [14]. In general it is not easy to decide whether the intersections of finitely generated subgroups of metabelian groups as a whole are finitely generated. Here we shall prove that there are algorithms which decide for a free metabelian group (or, more generally, for the wreath product of two free abelian groups) whether the intersection of two finitely generated subgroups is finitely generated or trivial. The proof of these results makes use of a new way of describing metabelian groups to which we now turn and which seems to be an essential element in understanding intersections.

1.2. Hybrid presentations of metabelian groups. As already noted in the abstract, even the intersection of two finitely generated free metabelian subgroups of a finitely generated free metabelian group need not be finitely generated. We shall give a number of examples of this and related phenomena in Section 5. In particular we find (Theorem 5.1) that the free metabelian group $F$ of rank 2 contains free metabelian subgroups $H_{1}$ and $H_{2}$ also of rank 2 with intersection $H_{1} \cap H_{2}=\left[H_{1}, H_{1}\right]=$ $\left[H_{2}, H_{2}\right]$. Thus their intersection is their derived group which is a free cyclic $\mathbb{Z} H_{i}$ module and hence free abelian of countably infinite rank. While this intersection is 
not finitely generated, it still has a description as a $\mathbb{Z} H_{i}$-module which is suitably finite. With this and other examples in mind, we introduce the notion of a hybrid generating system of a metabelian group.

Definition 1.1. Let $G$ be a metabelian group. A hybrid generating system of $G$ consists of

(1) an abelian normal subgroup $B$ of $G$ containing the derived group of $G$;

(2) a subset $X$ of $G$ which generates $G$ modulo $B$;

(3) a subset $Y$ of automorphisms of $B$ which generates an abelian subgroup $T$ of the automorphism group of $B$;

(4) a subset $Z$ of $B$ which generates $B$ viewed as a module over the integral group $\operatorname{ring} \mathbb{Z} T$ of $T$.

Such a hybrid generating system will be termed finite if $X, Y, Z$ are all finite.

The notion of a hybrid generating system gives rise in the obvious way to what we term a hybrid presentation.

Definition 1.2. A hybrid presentation of a metabelian group $G$ is

(1) a hybrid generating system $B, X, Y, Z$ of $G$, as above;

(2) a presentation of the abelian group $T$ on the generators $Y$;

(3) a presentation of $B$ as $\mathbb{Z} T$-module on the generators $Z$;

(4) a set of relations $R$ which induce a presentation of $G$ modulo $B$ and take the form $u_{\ell}=v_{\ell}$, where the $u_{\ell}$ are words in the set $X$ of $G$ and the $v_{\ell}$ are $\mathbb{Z} T$-module words in the set $Z$.

A hybrid presentation is termed finite if the sets $X, Y, Z$ of generators in the given hybrid generating system are finite and the corresponding sets of relations are finite.

Now if $G$ is a metabelian group and $B$ is an abelian normal subgroup of $G$ containing the derived group, then each element $g \in G$ defines an automorphism $\hat{g}$ of $B$ via conjugation:

$$
\hat{g}: b \mapsto g^{-1} b g(b \in B) .
$$

The mapping $\alpha$ which sends each element $g \in G$ to $\hat{g}$ is then a homomorphism of $G$ into the automorphism group of $B$ which induces a homomorphism $\alpha_{*}$ of $G / B$ into the automorphism group of $B$.

We observe that every finitely generated metabelian group $G$ has a finite hybrid presentation. We need first to show that it has a finite hybrid generating system. We choose $B$ to be the derived group of $G, X$ a set of elements of $G$ which generate it modulo its derived group, $T$ the subgroup of the automorphism group of $B$ generated by $Y=\left\{\alpha_{*}(x) \mid x \in X\right\}$ and, since the derived group of a finitely generated metabelian group is finitely generated as a module over the integral group ring of the factor derived group, $Z$ can be chosen to be any such set of generators. This provides 
us with a finite hybrid presentation of $G$ on choosing a finite presentation for $B$ as a $\mathbb{Z} T$-module and a finite set $R$ of relators which induce a presentation of $G$ modulo $B$ in the manner required.

As another example, observe that if $T$ is a finitely generated free abelian group and $B$ is a free $\mathbb{Z} T$ module with a finite basis, then setting $G=B, G$ has a finite hybrid presentation with $X=\emptyset, Y$ the free basis of $T$ and $Z$ the module free basis of $B$, and the set of relations $R=\emptyset$. Now, assuming $Y$ and $Z$ are non-empty, as an abelian group $B$ is free of countably infinite rank. So the same group can have numerous finite hybrid presentations of a very different character.

We remark that the data in a finite hybrid presentation allows one in principle to enumerate a recursive presentation of the underlying metabelian group $G$ as an abstract group.

Our interest in finite hybrid presentations is to use them in investigating the subgroups of given metabelian groups and to help understand how such subgroups intersect. Computing intersections of subgroups is often quite difficult. It turns out that the notion of a hybrid presentation is useful in this connection. To this end, we will need the following definition:

Definition 1.3. Let $G$ be a metabelian group given by a finite hybrid presentation as above. We term a (not necessarily finitely generated) subgroup $H$ of $G$ finitely hybrid-presentable if $H \cap B$ is finitely generated as a $P$-submodule of $B$ for some subgroup $P$ of $T$.

The data given in this definition allows us to find a finite hybrid presentation of $H$, which explains the terminology. Indeed choose $H \cap B$ to be the appropriate abelian normal subgroup of $H$ as required in Definition 1.1. Moreover, since $G / B$ is finitely generated, so too is $H / H \cap B$. Consequently the conditions laid down in Definition 1.1 can readily be satisfied. It follows that under these circumstances $H$ has a finite hybrid presentation. We shall make heavy use of this remark in the sequel. Furthermore, it is then not hard to see that a finitely generated subgroup $H$ of a finitely generated metabelian group is finitely hybrid-presentable (see Proposition 3.2). We have already observed that groups with a finite hybrid presentation need not be finitely generated.

It is important to note that this discussion of subgroups is relative in the sense that the subgroups here are viewed not in their own right, but as subgroups of the given containing group. For example, consider the free metabelian group $F$ of rank 3 with free generators $\left\{x_{1}, x_{2}, x_{3}\right\}$. Let $C$ be the normal closure of $\left[x_{1}, x_{2}\right]$ which is a free $\mathbb{Z}(F /[F, F])$-module. Consider the subgroup $K$ generated by $C$ and the cyclic group on $\left[x_{1}, x_{3}\right]$. Then $K$ is not a $\mathbb{Z} P$-submodule for any non-trivial subgroup $P \leq F /[F, F]$. Nor is it finitely generated as a module over the trivial subgroup. So as a subgroup $K$ is not finitely hybrid-presentable. As an abstract group, $K$ is a free abelian group of countably infinite rank, so in several different ways it does have a finite hybrid presentation. 
1.3. Intersections in certain wreath products. Our analysis of intersections of subgroups in free metabelian groups will apply more generally to certain wreath products. We will make extensive use of the Magnus embedding [16] of the free metabelian group into the wreath product $W=A$ wr $T$ of two finitely generated free abelian groups $A$ and $T$ with bases $\left\{a_{1}, \ldots, a_{m}\right\}$ and $\left\{t_{1}, \ldots, t_{n}\right\}$. Thus $W$ is the split extension $W=T \ltimes B$ where $B$ is the free $\mathbb{Z} T$-module with basis $\left\{a_{1}, \ldots, a_{m}\right\}$. Magnus showed that if $F$ is the free metabelian group with basis $\left\{x_{1}, \ldots, x_{n}\right\}$ and $m \geq n$ then the map defined by $x_{i} \mapsto t_{i} a_{i}$ is an embedding of $F$ into $W$.

The wreath product $W$ is of course finitely generated, and $W$ has a finite hybrid presentation as above using the base group $B$ as the abelian normal subgroup. We can now state our main results.

Theorem A. Let $W=A \mathrm{wr} T$ be the wreath product of finitely generated free abelian groups $A$ and $T$, and let $H_{1}$ and $H_{2}$ be finitely hybrid-presentable subgroups of $W$. Then $H_{1} \cap H_{2}$ is finitely hybrid-presentable. Moreover there is a uniform algorithm which, given finite hybrid presentations for $\mathrm{H}_{1}$ and $\mathrm{H}_{2}$, computes a finite hybrid presentation for $\mathrm{H}_{1} \cap \mathrm{H}_{2}$.

It follows from the proof of Theorem A that it is possible to describe when the intersection of two finitely generated subgroups of such wreath products is again finitely generated:

Theorem B. Let $W=A$ wr $T$ be the wreath product of finitely generated free abelian groups $A$ and $T$, and let $\pi$ denote the projection of $W$ onto $T$. Let $H_{1}$ and $\mathrm{H}_{2}$ be finitely generated subgroups of $W$ and let $H=H_{1} \cap H_{2}$. Then $H$ is finitely generated if and only if either $H \cap B=1$ or $H \pi$ has finite index in $H_{1} \pi \cap H_{2} \pi$.

As a consequence we can describe algorithms for testing whether such an intersection is finitely generated.

Corollary C. Let $W$ be the wreath product of two finitely generated free abelian groups. Let $H_{1}$ and $H_{2}$ be finitely generated subgroups of $W$. There is a uniform algorithm to determine whether or not $H_{1} \cap H_{2}$ is finitely generated, and, if so, whether or not $\mathrm{H}_{1} \cap \mathrm{H}_{2}$ is trivial.

Our methods depend heavily on the fact that the base group $B$ is a free $\mathbb{Z} T$ module. We leave open the question as to whether and how our results can be extended to finitely generated metabelian groups as a whole. Our notion of a finite hybrid presentation is available in the general case, but not all of our results carry over. In Section 5 we give an example showing that Theorem A does not carry over to finitely generated metabelian groups in general.

This paper is structured as follows. In Section 2 we set up some notation and review the necessary algorithmic background results. In Section 3 we prove some useful facts 
about the structure of finitely generated submodules of $B$ and of finitely generated subgroups of $W$. In Section 4.1 we show that the intersection $H_{1} \cap H_{2}$ of two subgroups of $W$ with finite hybrid presentations also has a finite hybrid presentation by showing that if $H_{i} \cap B$ is a finitely generated $P_{i}$-module, then $H_{1} \cap H_{2} \cap B$ is finitely generated as a $P_{1} \cap P_{2}$-module. We also show how to compute a finite set of module generators for $H_{1} \cap H_{2} \cap B$. In Section 4.2 we describe an algorithm to compute $\left(H_{1} \cap H_{2}\right) \pi$ when $H_{1}$ and $H_{2}$ are given by finite hybrid presentations, thus completing the calculation of the finite hybrid presentation for $H_{1} \cap H_{2}$ and the proof of Theorem A. In Section 4.3 we characterize those situations in which the intersection of two finitely generated subgroups is itself finitely generated (Theorem B and Corollary C). In Section 5 we construct a number of examples and use the above results to analyze their properties.

\section{Notation and algorithmic background}

If $G$ is a group, $\mathbb{Z} G$ denotes the group ring of $G$ over the ring $\mathbb{Z}$ of integers.

In $1954 \mathrm{P}$. Hall showed that finitely generated abelian-by-polycyclic groups (and hence finitely generated metabelian groups) satisfy max- $n$, the maximum condition for normal subgroups. Such a group $G$ is an extension of a normal abelian subgroup $A$ by a polycyclic group $P=G / A$. Hall showed that the group ring $\mathbb{Z} P$ is a right noetherian ring and $A$ is a finitely generated $\mathbb{Z} P$-module. This connection between commutative algebra and group theory has been very fruitful and has led to many finiteness conditions and algorithmic results.

As permanent notation we let $W$ denote the wreath product $W=A$ wr $T$ of two finitely generated free abelian groups $A$ and $T$ with bases $\left\{a_{1}, \ldots, a_{m}\right\}$ and $\left\{t_{1}, \ldots, t_{n}\right\}$. The projection of $W$ onto $T$ is denoted by $\pi$. We use multiplicative notation in our $\mathbb{Z} T$-modules, so an element $f \in \mathbb{Z} T$ acting on $b \in B$ gives $b^{f}$ (see below for an example of this notation).

Previously we noted that $W$ is the split extension $W=T \ltimes B$ where $B$ is the free $\mathbb{Z} T$-module with basis $\left\{a_{1}, \ldots, a_{m}\right\}$. But we will often view $B$ in a slightly different way. Namely $B$ is isomorphic to the restricted direct product of the groups $\left\{A^{t}=t^{-1} A t \mid t \in T\right\}$. To see this we observe that any element in the free module $B$ can be written in terms of monomials and hence gives a unique element in the direct product of the groups $\left\{A^{t} \mid t \in T\right\}$. For instance,

$$
a_{1}^{2-5 t_{1}^{-1} t^{2}} a_{2}^{3 t_{1}+t_{1}^{-1} t^{2}}=\left(a_{1}^{2}\right)\left(a_{2}^{3}\right)^{t_{1}}\left(a_{1}^{-5} a_{2}\right)^{t_{1}^{-1} t^{2}} .
$$

For $P \leq T$, we will use the term $P$-module for $\mathbb{Z} P$-module. If $S$ is a subset of the $P$-module $M$, we denote by $\bmod _{P}(S)$ the $P$-submodule of $M$ generated by $S$. Again we emphasize that we are using multiplicative notation in such $P$-modules.

Algorithmic questions are of fundamental interest in combinatorial group theory. In the 1950s it was shown that each of Dehn's fundamental decision problems - 
the word, conjugacy and isomorphism problems for finitely presented groups - is undecidable in general [19], [7]. Then in the mid-1980s it was shown [11], [5] that these problems are also unsolvable for finitely presented solvable groups of derived length at most 3.

The situation is dramatically different, however, if we further restrict to derived length at most 2, i.e. to metabelian groups. In 1959 P. Hall proved that finitely generated metabelian groups are residually finite [9]. Since finitely generated metabelian groups satisfy max- $n$, they are finitely presented in the variety of metabelian groups. It follows that the word problem for such groups is decidable (see, for example, Theorem 9.1.1 in [15]). In 1980 Romanovskii showed the problem of deciding membership in finitely generated subgroups of finitely generated metabelian groups is solvable. Then in 1982 Noskov proved that the conjugacy problem is also decidable in this context [18]. On the other hand, the decidability of the isomorphism problem for finitely presented metabelian groups remains unknown.

Many of the known algorithmic results for metabelian and related solvable groups are collected in the monograph [15], see particularly Sections 9.4 and 9.5. At the heart of many of these algorithms is the fact that the group ring $\mathbb{Z} P$ of a polycyclic group is submodule computable in the sense defined in [3]. This means that (1) $\mathbb{Z} P$ is a right Noetherian ring in which the ring operations are computable; (2) every finitely generated right $\mathbb{Z} P$-module $M$ is right Noetherian; and (3) there are algorithms which, when given a finite presentation of $M$, viewed as a $\mathbb{Z} P$-module, and a finite set $S$ of elements of $M$, find a presentation for $N=\bmod _{\mathbb{Z} P}(S)$ and decide membership in $N$.

For finitely generated metabelian groups in particular, Baumslag, Cannonito and Robinson [4] demonstrated the decidability of a host of additional natural problems. These include the computation of the derived subgroup, centralizers, the center, the Fitting subgroup and the Frattini subgroup. We will make extensive use of the results and methods of [3] and of [4].

Among the questions left open in [4] are two about the computation of intersections: given a finitely generated metabelian group $G$ and two finitely generated subgroups $H$ and $K$, can we decide if the intersection $H \cap K$ is finitely generated, and, if so, can we decide if $H \cap K$ is trivial? They show how to answer these questions when at least one of the subgroups, say $H$, is nearly normal, that is, when $H \cap[G, G]$ is normal in $G$. In this case $H \cap[G, G]$ is finitely generated as a $G /[G, G]$-module. Our results provide a rather different sort of answer in the context of free metabelian groups.

Subgroups with finite hybrid presentations, in our terminology, are similar to these nearly normal subgroups of [4]. Clearly nearly normal subgroups are finitely hybridpresentable in the case the subgroup $B$ in the definition is the derived group itself, that is, in case $B=[G, G]$. For completeness, we briefly explore the relationship between these two classes of subgroups in the case of the wreath product $W$.

Let $A$ be free abelian with basis $a_{1}, a_{2}, a_{3}$, let $T$ be free abelian with basis $t_{1}, t_{2}$, $t_{3}$, and let $W$ be the wreath product of $A$ and $T$. We begin by constructing a subgroup 
of $W$ which has a finite hybrid presentation but is not nearly normal. Let $H$ be the subgroup of $W$ generated by $t_{1} a_{1}$ and $t_{2} a_{2}$. It is easy to see directly that $H$ has a finite hybrid presentation: since $H B / B$ is free abelian with basis $\left\{t_{1} B, t_{2} B\right\}, H \cap B$ is generated as a normal subgroup of $H$ by the commutator $\left[t_{1} a_{1}, t_{2} a_{2}\right]$; therefore, $H \cap B=H \cap[W, W]$, and it is finitely generated as a $\left\langle t_{1}, t_{2}\right\rangle$-module. On the other hand, $H$ is not nearly normal since $H \cap[W, W]$ is not normal in $W$.

Next we construct a subgroup $K$ of $W$ which is nearly normal, but viewed as a subgroup of $W$, does not have a finite hybrid presentation. Let $C$ be the $T$-module generated by $\left[t_{1} a_{1}, t_{2} a_{2}\right]$. Let $K$ be the subgroup of $W$ generated by $a_{3}$ and $C$. It is not hard to see that $K=K \cap B$ is not finitely generated as a $P$-submodule for any $P \leq T$ : the only element $t$ of $T$ such that $K^{t} \leq K$ is $t=1$, so $K$ is a $P$ submodule if and only if $P=1$, but $K$ is not finitely generated as an abelian group. Thus we see that $K$ does not have a finite hybrid presentation. On the other hand, $K \cap[W, W]=C$, and $C$ is finitely generated as a $W /[W, W]$-module, so $K$ is nearly normal.

\section{About finite generation}

Here we will be concerned with $W=A$ wr $T$, where $A$ and $T$ are as usual finitely generated free abelian groups on $\left\{a_{1}, \ldots, a_{m}\right\}$ and $\left\{t_{1}, \ldots, t_{n}\right\}$ and $B$ is the base group of $W$.

In this section we prove structural theorems about finitely generated modules (not necessarily submodules) contained in $B$ and finitely generated subgroups of $W$.

For $d \in B$, the support of $d$, denoted by $\sigma(d)$, is the set of all elements $t \in T$ such that the image of $d$ under the projection from $B$ to $A^{t}$ is non-trivial. Note that $\sigma(d)$ is always finite. If $M$ is a subgroup of $B$ then we put $\sigma(M)=\bigcup_{d \in M} \sigma(d)$. Observe that if $M_{1}$ and $M_{2}$ are subgroups of $B$, then $\sigma\left(M_{1} M_{2}\right)=\sigma\left(M_{1}\right) \cup \sigma\left(M_{2}\right)$. If $d \in B$ and $P$ is a subgroup of $T$ then the support of the cyclic $P$-module $d^{\mathbb{Z} P}$ is $\sigma(d) P$. Also $\sigma\left(M_{1} \cap M_{2}\right) \subseteq \sigma\left(M_{1}\right) \cap \sigma\left(M_{2}\right)$.

We begin with a lemma characterizing finitely generated modules of $B$ in terms of supports.

Lemma 3.1. Let $P$ be a subgroup of $T$, and let $M \leq B$ be a $P$-module. Then $M$ is finitely generated as a $P$-module if and only if there exists a finite subset $S$ of $T$ such that $\sigma(M) \subseteq\{s p \mid s \in S, p \in P\}$. If $M$ is generated by $m_{1}, m_{2}, \ldots, m_{k}$ as a $P$-module and $S=\bigcup_{i=1}^{i=k} \sigma\left(m_{i}\right)$, then $\sigma(M)=\{s p \mid s \in S, p \in P\}$.

Proof. Suppose that $M$ is generated as a $P$-module by $m_{1}, \ldots, m_{k}$. Let $S$ be $\bigcup_{i=1}^{i=k} \sigma\left(m_{i}\right)$. For $p \in P$, since $\sigma\left(m_{i}^{p}\right)=\sigma\left(m_{i}\right) p$, we see that $\{s p \mid s \in S$, $p \in P\} \subseteq \sigma(M)$. To see that $\sigma(M) \subseteq\{s p \mid s \in S, p \in P\}$, suppose that $m \in M$. Then $m$ can be written as a product of elements of the form $b^{p}$, where $b=m_{i}^{\epsilon}$ 
for some $i$, some $\epsilon= \pm 1$, and some $p \in P$. Now $\sigma\left(b^{p}\right) \subset S P$, and therefore $\sigma(m) \subset S P$.

For the converse, suppose that there exists a finite subset $S$ of $T$ such that $\sigma(M) \subseteq$ $\{s p \mid s \in S, p \in P\}$. Let $C \leq B$ be the direct product of the subgroups $\left\{A^{s p} \mid s \in\right.$ $S, p \in P\}$. Notice that $C$ is a $P$-module. Let $a_{1}, \ldots, a_{n}$ be a set of generators for $A$ as an abelian group. $C$ is finitely generated as a $P$-module by

$$
\bigcup_{s \in S}\left\{a_{1}^{s}, \ldots, a_{n}^{s}\right\} .
$$

Therefore, $C$ is a Noetherian $P$-module. Since $M$ is a $P$-submodule of $C, M$ is finitely generated.

The next proposition characterizes finitely generated subgroups of $W$ in terms of their intersection with $B$. Here, as before, $\pi$ is the projection of $W$ onto $T$.

Proposition 3.2. Let $H$ be a subgroup of $G$ and let $P=H \pi$. Then $H \cap B$ is a $P$-module and $H$ is finitely generated if and only if $H \cap B$ is finitely generated as a $P$-module.

Proof. Suppose $H$ is finitely generated. Since $H /(H \cap B)$ is finitely presented, $H \cap B$ is finitely generated as a normal subgroup of $H$. Therefore, $H \cap B$ is finitely generated as a $P$-module.

Conversely, suppose that $H \cap B$ is generated as a $P$-module by $d_{1}, \ldots, d_{r}$. Let $h_{1}, \ldots, h_{s}$ be elements of $H$ such that $h_{1}(H \cap B), \ldots, h_{s}(H \cap B)$ generate $H /(H \cap B)$ as an abelian group. Then $d_{1}, \ldots, d_{r}, h_{1}, \ldots, h_{s}$ generate $H$ as a group.

We want to be able to decide whether a subgroup given by a finite hybrid presentation is finitely generated. We begin with an elementary observation about intersections of subgroups of finitely generated abelian groups.

Lemma 3.3. Let $H$ and $K$ be subgroups of $T$. Suppose that $H$ is a subset of the union of finitely many cosets of $K$. Then $[H: H \cap K]<\infty$.

Proof. Let $h \in H$. Let $\left\{g_{1} K, g_{2} K, \ldots, g_{r} K\right\}$ be a set of distinct cosets whose union contains $H$. By the pigeonhole principle, there exist positive integers $0<\beta<$ $\alpha \leq r+1$ and a $g_{i}$ such that $h^{\alpha} \in g_{i} K$ and $h^{\beta} \in g_{i} K$. Therefore $h^{\alpha-\beta} \in K$. Therefore, $H /(H \cap K)$ is a finitely generated abelian group of finite exponent, and hence $[H: H \cap K]<\infty$.

Now we can recognize when a subgroup with a finite hybrid presentation is actually finitely generated.

Proposition 3.4. Suppose that $H \cap B$ is finitely generated as a $P$-module for some $P \leq T . H$ is finitely generated if and only if $H \cap B=1$ or $[P: H \pi \cap P]<\infty$. 
Proof. First suppose that $H$ is finitely generated. Then $H \cap B$ is finitely generated as an $H \pi$-module and $H \cap B$ is also finitely generated as a $P$-module. Therefore, by Proposition 3.1 there exist finite subsets $R$ and $S$ of $T$ such that $\sigma(H \cap B)=$ $R P=S H \pi$. Fix $r \in R$. For all $p \in P$, there exist $s \in S$ and $q \in H \pi$ such that $s q=r p$, so $p=r^{-1} s q$. Thus, $P$ is a subset of the union of finitely many cosets of $H \pi$. By Lemma 3.3, $[P: H \pi \cap P]<\infty$.

Conversely, suppose that $[P: P \cap H \pi]<\infty$ and $H \cap B \neq 1$. Since $H \cap B$ is finitely generated as a $P$-module, it is also finitely generated as a $P \cap H \pi$-module, and hence as an $H \pi$-module. By Proposition 3.2, $H$ is finitely generated as a subgroup.

\section{Intersections}

In this section we prove that, in the wreath product $W$, the intersection of two subgroups with finite hybrid presentations also has a finite hybrid presentation, and we describe an algorithm to find such a description. This, together with Proposition 3.4, shows that we can decide whether or not the intersection of two subgroups with finite hybrid presentations is finitely generated, and, if so, if it is trivial.

4.1. The structure of $H_{1} \cap H_{2} \cap B$. Our first proposition is about intersections of submodules of $B$.

Proposition 4.1. Let $P_{1}$ and $P_{2}$ be subgroups of $T$. Suppose that $K_{i}$ is a finitely generated $P_{i}$-submodule of $B$ for $i=1,2$. Then $K_{1} \cap K_{2}$ is finitely generated as a $P_{1} \cap P_{2}$-module.

Proof. Let $K=K_{1} \cap K_{2}$. $K$ is a $\left(P_{1} \cap P_{2}\right)$-module.

By Lemma 3.1 it suffices to show that there exists a finite subset $U$ of $T$ such that

$$
\sigma(K) \subseteq\left\{s p \mid s \in U, p \in P_{1} \cap P_{2}\right\} .
$$

By Lemma 3.1 we know that there exists a finite set $R$ of $T$ such that $\sigma\left(K_{1}\right)=$ $R P_{1}$. Likewise, there exists a finite set $S$ of $T$ such that $\sigma\left(K_{2}\right)=S P_{2}$. Since $\sigma(K) \subseteq \sigma\left(K_{1}\right) \cap \sigma\left(K_{2}\right)$, it suffices to show that there exists a finite subset $U$ of $T$ such that $R P_{1} \cap S P_{2} \subseteq U\left(P_{1} \cap P_{2}\right)$.

Let $R=\left\{r_{1}, \ldots, r_{a}\right\}$ and let $S=\left\{s_{1}, \ldots, s_{b}\right\}$. Let $I$ be the set of those ordered pairs $(i, j)$ of indices for which there exist $p \in P_{1}$ and $q \in P_{2}$ such that $r_{i} p=s_{j} q$. For $(i, j) \in I$, let $p_{i, j} \in P_{1}$ and $q_{i, j} \in P_{2}$ be one such solution, so $r_{i} p_{i, j}=s_{j} q_{i, j}$. Let

$$
U=\left\{r_{i} p_{i, j} \mid(i, j) \in I\right\} .
$$

Let $t \in R P_{1} \cap S P_{2}$. Then there exists $(i, j) \in I, p \in P_{1}, q \in P_{2}$ such that $t=r_{i} p=s_{j} q$. If we put $z=\left(p_{i, j}\right)^{-1} p$ then computing in the abelian group $T$ we 
have

$$
z=\left(p_{i, j}\right)^{-1} p=\left(r_{i} p_{i, j}\right)^{-1} r_{i} p=\left(s_{j} q_{i, j}\right)^{-1} s_{j} q=q_{i, j}^{-1} q .
$$

Therefore $p_{i, j} p^{-1} \in P_{1} \cap P_{2}$, so $t=r_{i} p_{i, j} z$ where $z=\left(p_{i, j}\right)^{-1} p \in P_{1} \cap P_{2}$.

As a consequence we have the following result which is the first assertion of Theorem A. The algorithmic assertions of Theorem A will be established in Propositions 4.7 and 4.12 below.

Corollary 4.2 ( = first part of Theorem A). If $H_{1}$ and $H_{2}$ are subgroups of $W$ with finite hybrid presentations, then $\mathrm{H}_{1} \cap \mathrm{H}_{2}$ also has a finite hybrid presentation.

Proof. Let $P_{1}, P_{2}$ be subgroups of $T$ such that $H_{i} \cap B$ is finitely generated as a $P_{i}$-module. Then $H_{1} \cap H_{2} \cap B=\left(H_{1} \cap B\right) \cap\left(H_{2} \cap B\right)$, which is finitely generated as a $P_{1} \cap P_{2}$-module by Proposition 4.1 .

In order to show that we can actually compute $H_{1} \cap H_{2} \cap B$ we will need to generalize Lemma 2.2 of [3] which states that if $M$ is a finitely generated $P$-module, and $M_{1}$ and $M_{2}$ are finitely generated $P$-submodules of $M$, then we can compute $M_{1} \cap M_{2}$. In particular we will need to be able to compute $M_{1} \cap M_{2}$ when each $M_{i}$ is a $P_{i}$-module, but $P_{1} \neq P_{2}$. Lemma 4.6 will show that this is possible when $M_{1}$ and $M_{2}$ are submodules of $B \leq W$; Lemmas 4.3, 4.4 and 4.5 will pave the way.

Lemma 4.3. Let $G$ be a finitely generated abelian group, and let $H$ be a subgroup of $G$. Let $F$ be a free $G$-module with finite basis $\mathcal{B}$. Let $S$ be a finite subset of $F$. There is a uniform algorithm to compute a finite set of $H$-module generators for $\bmod _{H}(\mathscr{B}) \cap \bmod _{G}(S)$.

Proof. This is a special case of Corollary 2.13 of [3].

Lemma 4.4. Let $P \leq T$, and let $U=\left\{u_{1}, u_{2}, \ldots, u_{n}\right\}$ be a set of elements of $T$ such that if $i \neq j$, then $u_{i} P \neq u_{j} P$. Then $U$ is a basis for the free $P$-submodule $N$ of $\mathbb{Z} T$ given by $N=\bmod _{P} U$. Thus, $A^{U P}$ is a free $P$-submodule with basis $\left\{a^{u} \mid a \in \mathcal{A}, u \in \mathcal{U}\right\}$, where $\mathcal{A}$ is a basis for $A$ as an abelian group.

Proof. Suppose that $f_{1}, f_{2}, \ldots, f_{n} \in \mathbb{Z} P$ such that $u_{1} f_{1}+u_{2} f_{2}+\cdots+u_{n} f_{n}=0$. Notice that $\sigma\left(u_{i} f_{i}\right) \subset u_{i} P$, so if $i \neq j$, then $\sigma\left(u_{i} f_{i}\right)$ is disjoint from $\sigma\left(u_{j} f_{j}\right)$. From this it follows that $u_{i} f_{i}=0$ for all $i=1,2, \ldots, n$. Now $u_{i} \in T$ so $u_{i} \neq 0$ : remember, we are using multiplicative notation in $T$. Since $\mathbb{Z} T$ is an integral domain, it follows that $f_{i}=0$ for $i=1,2, \ldots, n$. This shows that $N$ is free with basis $U$.

It is easy to see that since $U$ is a basis for a free $P$-submodule $N$ of $\mathbb{Z} T$, then $A^{N}=A^{U P}$ is a free $P$-submodule of $B$ with basis $\left\{a^{u} \mid a \in \mathcal{A}, u \in U\right\}$. 
Lemma 4.5. Let $W$ be the wreath product of finitely generated free abelian groups $A$ and $T$. Let $P$ and $P_{1}$ be subgroups of $T$ such that $P \leq P_{1}$. Let $M_{1}$ be a finitely generated $P_{1}$-submodule of $B$. Let $U$ be a finite subset of $T$ such that if $u, v \in U$, then $u P_{1} \neq v P_{1}$. Then there is a uniform algorithm to compute a finite set of generators for $M_{1} \cap \bmod _{P} A^{U}$ as a P-module.

Proof. Let $Q=U P_{1} \cup \sigma\left(M_{1}\right)$. By Lemma 3.1, $\sigma\left(M_{1}\right)$ is the union of finitely many cosets of $P_{1}$. Therefore, $Q$ is itself the union of finitely many cosets of $P_{1}$. Thus there exists a finite subset $\mathcal{Q} \supseteq U$ of $T$ such that $Q=\bigcup_{u \in \mathcal{Q}} u P_{1}$ and $u, v \in \mathbb{Q}$, $u \neq v$ implies that $u P_{1} \neq v P_{1}$. Let $\mathcal{C}=\left\{a^{q} \mid a \in \mathcal{A}, q \in \mathcal{Q}\right\}$. By Lemma $4.4 A^{Q}$ is free as a $P_{1}$-module and $\mathcal{C}$ is a basis for $A^{Q}$ as such.

Let $N$ be the $P$-module given by $N=\bmod _{P} C \cap M_{1}$. By Lemma 4.3 we can find generators for $N$ as a $P$-module. By Lemma 2.2 of [3] we can then compute $N^{\prime}=N \cap \bmod _{P} A^{U}$ as a $P$-module.

We will now show that $N^{\prime}=M_{1} \cap \bmod _{P} A^{U}$. Since $U \subseteq \mathcal{Q}, \bmod _{P} A^{U} \subseteq$ $\bmod _{P} \ell$. Therefore $M_{1} \cap \bmod _{P} A^{U} \subseteq M_{1} \cap \bmod _{P} \ell=N$ and hence $M_{1} \cap$ $\bmod _{P} A^{U} \subseteq N^{\prime}$. On the other hand, $N^{\prime} \subseteq N \subseteq M_{1}$, and clearly $N^{\prime} \subseteq \bmod _{P} A^{U}$. Therefore $N^{\prime} \subseteq M_{1} \cap \bmod _{P} A^{U}$.

Lemma 4.6. Let $W$ be the wreath product of finitely generated free abelian groups $A$ and $T$. For $i=1,2$, let $P_{i}$ be a subgroup of $T$, and let $P=P_{1} \cap P_{2}$. Let $M_{i}$ be a finitely generated $P_{i}$-submodule of $B$ and let $M$ be a finitely generated $P$-module such that $M \geq M_{1} \cap M_{2}$. Then there is a uniform algorithm to compute a finite set of generators for $M_{1} \cap M_{2}$ as a $P$-module.

Proof. It suffices to describe an algorithm to compute $P$-module generators for $M \cap M_{1}$ since $M_{1} \cap M_{2}=\left(M \cap M_{1}\right) \cap\left(M \cap M_{2}\right)$. The difficulty arises because $M_{1}$ may not be finitely generated as a $P$-module. In the proof of Proposition 3.1, we see that we can compute a finite subset $U$ of $T$ such that $\sigma\left(M_{1} \cap M\right) \subseteq U P$ and $u, v \in U, u \neq v$ implies $u P \neq v P$.

Let $N=\bmod _{P} A^{U}$. Then $M_{1} \cap M \subseteq N$. It is sufficient to find a finite set of generators for $N \cap M_{1}$ as a $P$-module, since we can then compute $M \cap M_{1}=$ $M \cap\left(N \cap M_{1}\right)$. Lemma 4.5 suffices for computing $N \cap M_{1}$.

The next result provides one of the algorithms needed for Theorem A. The other required algorithm is given by Proposition 4.12 below.

Proposition 4.7. Let $W$ be the wreath product of finitely generated free abelian groups $A$ and $T$ and let $H_{1}$ and $H_{2}$ be subgroups of $W$ given by finite hybrid presentations. There is a uniform algorithm to compute a finite generating set for $\mathrm{H}_{1} \cap \mathrm{H}_{2} \cap \mathrm{B}$ as an $H_{1} \pi \cap H_{2} \pi$-module.

Proof. Suppose that $H_{i} \cap B$ is given as a $P_{i}$-module. Let $P=P_{1} \cap P_{2}$. Our first task to find a finite set $U \subseteq T$ such that $H_{1} \cap H_{2} \cap B$ is contained in the 
$P$-module generated by the direct sum of $\left\{A^{u} \mid u \in U\right\}$. Examination of the proof of Proposition 4.1 shows that given elements $r, s \in T$, we must be able to decide if there exist elements $p_{1} \in P_{1}$ and $p_{2} \in P_{2}$ such that $r p_{1}=s p_{2}$, and to find such elements if they exist. Since $r p_{1}=s p_{2}$ if and only if $r s^{-1}=p_{1}^{-1} p_{2}$, we can use linear algebra to test whether or not $r s^{-1}$ is an element of $P_{1} P_{2}$. If it is, we can enumerate the elements of $P_{1}$ and $P_{2}$ until we find $p_{1} \in P_{1}$ and $p_{2} \in P_{2}$ such that $r s^{-1}=p_{1}^{-1} p_{2}$. By Lemma 4.6 we can then compute a finite generating set for $H_{1} \cap H_{2} \cap B$.

4.2. The structure of $\left(\boldsymbol{H}_{1} \cap \boldsymbol{H}_{2}\right) \pi$. Suppose we are given finite hybrid presentations for subgroups $H_{1}$ and $H_{2}$ of $W$. The finite hybrid presentation for $H_{i}$ consists of a finite set of elements of $H_{i}$ whose images generate $H_{i} B / B$, a finite set of elements of $T$ that generate a subgroup we call $P_{i}$, and a finite set of elements of $H_{i}$ that generate $H_{i} \cap B$ as a $P_{i}$-module. In order to complete our calculation of a finite hybrid presentation for $H_{1} \cap H_{2}$, we must compute $\left(H_{1} \cap H_{2}\right) \pi$. Let $Q_{i}=H_{i} B / B$. Note that while $H_{i} \cap B$ is also a $Q_{i}$-module, it may not be finitely generated as such.

We examine three cases:

(1) $B \leq H_{2}$;

(2) $Q_{1}=Q_{2}$;

(3) the general case.

Case 1 is easy: if $B \leq H_{2}$, then $\left(H_{1} \cap H_{2}\right) B=H_{1} B \cap H_{2} B$, so computations in $W / B$ suffice to find generators for $\left(H_{1} \cap H_{2}\right) B / B$.

It is also easy to see that the general case (3) can be handled assuming that cases 1 and 2 can be handled. To see this, let $K=H_{1} B \cap H_{2} B$. Computations in $W / B$ suffice for computing $K$. Let $H_{i}^{\prime}=H_{i} \cap K$. Using case 1 we compute generators for $H_{i}^{\prime} B / B$. Now $H_{1}^{\prime} \cap H_{2}^{\prime}=H_{1} \cap H_{2}$. Furthermore,

$$
H_{1}^{\prime} B=\left(H_{1} \cap\left(H_{1} B \cap H_{2} B\right)\right) B=\left(H_{1} \cap H_{2} B\right) B=H_{1} B \cap H_{2} B .
$$

Thus $H_{1}^{\prime} B=H_{2}^{\prime} B$, so we use case 2 to compute $\left(H_{1}^{\prime} \cap H_{2}^{\prime}\right) B / B=\left(H_{1} \cap H_{2}\right) B / B$ as required.

We are left with case 2, in which $Q_{1}=Q_{2}$. Let $Q=Q_{1}$ (so $Q=Q_{2}$ as well). Notice that $H_{1} \cap B$ and $H_{2} \cap B$ are both $Q$-modules, though they might not be finitely generated as such.

Our primary task will be to find generators for the following subgroup $P$ of $Q$ :

$$
P=\left\{p \in Q \mid \text { there exists } a \in B \text { such that } p a \in H_{1} \cap H_{2}\right\} .
$$

We will now mimic the proof of Theorem 5.6 of [4] by describing $P$ as the kernel of a derivation $\delta$ from $Q$ to a quotient of a certain $Q$-submodule of $B$. We will do so in several steps.

In this case there exist sets $S_{1} \subseteq H_{1}$ and $S_{2} \subseteq H_{2}$ of the form

$$
S_{1}=\left\{x_{1} b_{1}, x_{2} b_{2}, \ldots, x_{r} b_{r}\right\}, \quad S_{2}=\left\{x_{1} c_{1}, x_{2} c_{2}, \ldots, x_{r} c_{r}\right\},
$$


where the $x_{i}$ 's form a basis for $Q$ as a free abelian group, the $b_{i}$ 's and $c_{i}$ 's are in $B$, and where the images of $S_{i}$ generate $H_{i} B / B$.

Let $\theta_{i}$ be the map that takes $m \in \mathbb{Z}$ to $f \in \mathbb{Z} Q$ defined as follows: for any $b \in B,\left(x_{i} b\right)^{m}=x_{i}^{m} b^{f}=x_{i}^{m} b^{m \theta_{i}}$. For $m>0$, one can check that $m \theta_{i}=$ $1+x_{i}+\cdots+x_{i}^{m-1}$ and that $(-m) \theta_{i}=-x_{i}^{-1}-x_{i}^{-2}-\cdots-x_{i}^{-m}$. Consequently, for all $m \in \mathbb{Z}$, the identity $\left(x_{i}-1\right)\left(m \theta_{i}\right)=\left(x_{i}^{m}-1\right)$ holds. We also define the elements

$$
d_{i}=\left(x_{i} c_{i}\right)^{-1}\left(x_{i} b_{i}\right)=b_{i} c_{i}^{-1}
$$

for $i=1,2, \ldots, r$, which measure the difference between the corresponding generators of the $S_{i}$.

\section{Lemma 4.8.}

$$
\begin{aligned}
& \left(x_{1} b_{1}\right)^{v_{1}}\left(x_{2} b_{2}\right)^{v_{2}} \ldots\left(x_{r} b_{r}\right)^{v_{r}}
\end{aligned}
$$

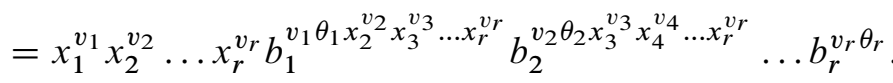

Similar equations hold for the $c_{i}$ and $d_{i}$.

Proof.

$$
\begin{aligned}
& \left(x_{1} b_{1}\right)^{v_{1}}\left(x_{2} b_{2}\right)^{v_{2}} \ldots\left(x_{r} b_{r}\right)^{v_{r}} \\
& =\left(x_{1}^{v_{1}} b_{1}^{v_{1} \theta_{1}}\right)\left(x_{2}^{v_{2}} b_{2}^{v_{2} \theta_{2}}\right) \ldots\left(x_{r}^{v_{r}} b_{r}^{v_{r} \theta_{r}}\right)
\end{aligned}
$$

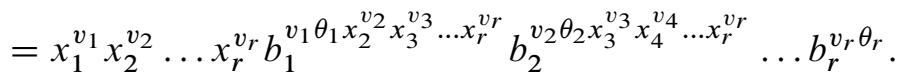

We now define a set map $\mu$ from $Q$ to $B$ by

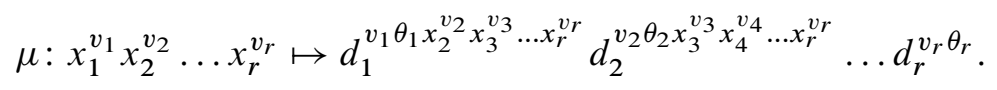

Notice that by Lemma 4.8, if $q=x_{1}^{v_{1}} x_{2}^{v_{2}} \ldots x_{r}^{v_{r}}$, then

$$
q \mu=q^{-1}\left(x_{1} d_{1}\right)^{v_{1}}\left(x_{2} d_{2}\right)^{v_{2}} \ldots\left(x_{r} d_{r}\right)^{v_{r}} .
$$

That is, if we substitute $x_{i} d_{i}$ for $x_{i}$ in $q$ and express the result in the form $q b$ with $b \in B$, then $b=q \mu$. Alternatively, if we use functional notation $q=q\left(x_{i}\right)$ and $q\left(x_{i} b_{i}\right)$ and $q\left(x_{i} c_{i}\right)$ are the results of replacing the $x_{i}$ by $x_{i} b_{i}$ and $x_{i} c_{i}$ respectively, then $q \mu=q\left(x_{i} c_{i}\right)^{-1} q\left(x_{i} b_{i}\right)$. The following proposition gives a key property of this map $\mu$ for intersections.

Proposition 4.9. $q \in P$ if and only if $q \mu \in\left(H_{1} \cap B\right)\left(H_{2} \cap B\right)$. 
Proof. We have the following chain of equivalences:

$x_{1}^{v_{1}} x_{2}^{v_{2}} \ldots x_{r}^{v_{r}} \in P$

$\Longleftrightarrow \exists a \in B$ such that $x_{1}^{v_{1}} x_{2}^{v_{2}} \ldots x_{r}^{v_{r}} a \in H_{1} \cap H_{2}$

$\Longleftrightarrow \exists a \in B$ such that

$$
\begin{aligned}
& \left(x_{1}^{v_{1}} x_{2}^{v_{2}} \ldots x_{r}^{v_{r}} a\right)^{-1}\left(x_{1} b_{1}\right)^{v_{1}}\left(x_{2} b_{2}\right)^{v_{2}} \ldots\left(x_{r} b_{r}\right)^{v_{r}} \in H_{1} \cap B \\
& \text { and }\left(x_{1}^{v_{1}} x_{2}^{v_{2}} \ldots x_{r}^{v_{r}} a\right)^{-1}\left(x_{1} c_{1}\right)^{v_{1}}\left(x_{2} c_{2}\right)^{v_{2}} \ldots\left(x_{r} c_{r}\right)^{v_{r}} \in H_{2} \cap B
\end{aligned}
$$

$\Longleftrightarrow \exists a \in B, m_{1} \in H_{1} \cap B, m_{2} \in H_{2} \cap B$ such that

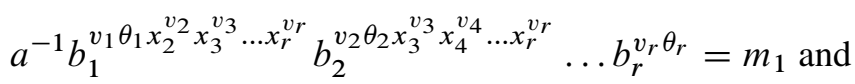

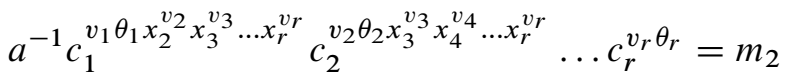

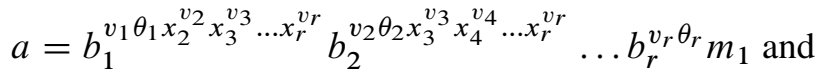

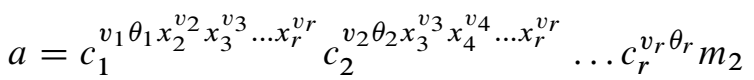

$\Longleftrightarrow \exists m_{1} \in H_{1} \cap B, m_{1} \in H_{2} \cap B$ such that

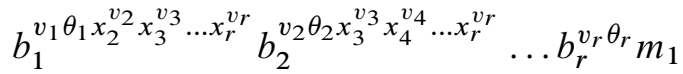

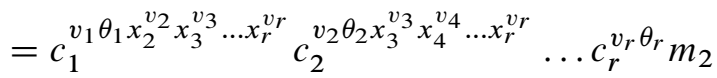

$\Longleftrightarrow \exists m_{1} \in H_{1} \cap B, m_{2} \in H_{2} \cap B$ such that

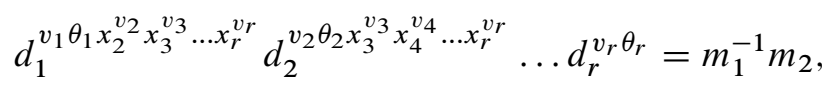

which is the desired result.

Lemma 4.10. For all $i, j=1,2, \ldots, r,\left[x_{i} d_{i}, x_{j} d_{j}\right] \in\left(H_{1} \cap B\right)\left(H_{2} \cap B\right)$.

Proof. Since $T$ is abelian and so $\left[x_{i}, x_{j}\right]=1$, an easy calculation gives

$$
\left[x_{i} d_{i}, x_{j} d_{j}\right]=d_{i}^{-1} x_{i}^{-1} d_{j}^{-1} x_{j}^{-1} x_{i} d_{i} x_{j} d_{j}=d_{i}^{x_{j}-1} d_{j}^{1-x_{i}} .
$$

We get a similar equation by substituting $b$ 's for $d$ 's, and another by substituting $c$ 's for $d$ 's. Since $d_{i}^{x_{j}-1} d_{j}^{1-x_{i}}=b_{i}^{x_{j}-1} b_{j}^{1-x_{i}}\left(c_{i}^{x_{j}-1} c_{j}^{1-x_{i}}\right)^{-1},\left[x_{i} d_{i}, x_{j} d_{j}\right]=$ $\left[x_{i} b_{i}, x_{j} b_{j}\right]\left(\left[x_{i} c_{i}, x_{j} c_{j}\right]\right)^{-1} \in\left(H_{1} \cap B\right)\left(H_{2} \cap B\right)$.

Let $M$ be the $Q$-submodule of $B$ generated by the $d_{i}$ 's and the $Q$-module $\left(H_{1} \cap B\right)\left(H_{2} \cap B\right)$. Let $\delta$ be the map from $Q$ to $M /\left(H_{1} \cap B\right)\left(H_{2} \cap B\right)$ given by $q \delta=q \mu\left(H_{1} \cap B\right)\left(H_{2} \cap B\right)$.

Lemma 4.11. $\delta$ is a derivation. 
Proof. Let $p=x_{1}^{u_{1}} x_{2}^{u_{2}} \ldots x_{r}^{u_{r}}$ and let $q=x_{1}^{v_{1}} x_{2}^{v_{2}} \ldots x_{r}^{v_{r}}$. Then modulo $\left(H_{1} \cap B\right)\left(H_{2} \cap B\right)$ we have the congruences

$$
\begin{aligned}
(p q) \delta & \equiv(p q)^{-1}\left(x_{1} d_{1}\right)^{u_{1}+v_{1}}\left(x_{2} d_{2}\right)^{u_{2}+v_{2}} \ldots\left(x_{r} d_{r}\right)^{u_{r}+v_{r}} \\
& \equiv(p q)^{-1}\left(x_{1} d_{1}\right)^{u_{1}}\left(x_{1} d_{1}\right)^{v_{1}} \ldots\left(x_{r} d_{r}\right)^{u_{r}}\left(x_{r} d_{r}\right)^{v_{r}} \\
& \equiv(p q)^{-1}\left(x_{1} d_{1}\right)^{u_{1}} \ldots\left(x_{r} d_{r}\right)^{u_{r}}\left(x_{1} d_{1}\right)^{v_{1}} \ldots\left(x_{r} d_{r}\right)^{v_{r}} \\
& \equiv\left(p^{-1}\left(x_{1} d_{1}\right)^{u_{1}} \ldots\left(x_{r} d_{r}\right)^{u_{r}}\right)^{q} q^{-1}\left(x_{1} d_{1}\right)^{v_{1}} \ldots\left(x_{r} d_{r}\right)^{v_{r}} \\
& \equiv(p \delta)^{q}(q \delta),
\end{aligned}
$$

which means that $(p q) \delta \equiv(p \delta)^{q}(q \delta) \bmod \left(H_{1} \cap B\right)\left(H_{2} \cap B\right)$. (Notice the use of Lemma 4.10 in line 3.)

This brings us to the following result which is the remaining algorithm needed for Theorem A. Taken together, Corollary 4.2 and Propositions 4.7 and 4.12 establish Theorem A.

Proposition 4.12. Let $W=A$ wr $T$ be the wreath product of finitely generated free abelian groups $A$ and $T$, and let $H_{1}$ and $H_{2}$ be subgroups of $W$ given by finite hybrid presentations, where $H_{i} \cap B$ is given as a $P_{i}$-module. There is a uniform algorithm to compute a finite set of generators for $\left(H_{1} \cap H_{2}\right) \pi$.

Proof. As we saw at the start of this section, we may assume that $H_{1} \pi=H_{2} \pi$. Let $Q=H_{1} \pi$. Let $\delta$ be the derivation from $Q$ to $M /\left(H_{1} \cap B\right)\left(H_{2} \cap B\right)$ defined above. By Proposition 4.9, the kernel of $\delta$ is $P$. Clearly, $M /\left(H_{1} \cap B\right)\left(H_{2} \cap B\right)$ is finitely generated as a $Q$-module, so by Lemma 5.5 of [4], we can compute $P$. Since membership testing in $H_{1} \cap H_{2}$ is possible, for each generator $p$ of $P$ we can do an exhaustive enumeration search to find an element $b$ of $B$ such that $p b \in H_{1} \cap H_{2}$. The images in $W \pi$ of the elements $p b \in H_{1} \cap H_{2}$ so obtained generate $\left(H_{1} \cap H_{2}\right) \pi$.

4.3. Finite generation of intersections. Notice that $\left(H_{1} \cap H_{2}\right) \pi \leq H_{1} \pi \cap H_{2} \pi$, but the reverse inequality does not necessarily hold; indeed, $\left(H_{1} \cap H_{2}\right) \pi$ may not have finite index in $H_{1} \pi \cap H_{2} \pi$. The proposition below shows that this fact is at the heart of why the intersection of two finitely generated subgroups of $W$ may not itself be finitely generated.

Theorem 4.13 (= Theorem B). Let $H_{1}$ and $H_{2}$ be finitely generated subgroups of $W$ and let $H=H_{1} \cap H_{2}$. Then $H$ is finitely generated if and only if either $H \cap B=1$ or $H \pi$ has finite index in $H_{1} \pi \cap H_{2} \pi$.

Proof. Let $K=H \cap B$. By Proposition $3.2 H$ is finitely generated if and only if $K$ is finitely generated as an $H \pi$-module. 
Suppose that $H$ is finitely generated and that $K \neq 1$. By Lemma 3.1 there exists a nonempty finite subset $S$ of $T$ such that $\sigma(K) \subseteq\{s p \mid s \in S, p \in H \pi\}$. But $K$ is a $\left(H_{1} \pi \cap H_{2} \pi\right)$-module, so $\sigma(K)$ must be closed under right multiplication by $H_{1} \pi \cap H_{2} \pi$. It follows that $H \pi$ must have finite index in $H_{1} \pi \cap H_{2} \pi$.

Clearly, if $K=1$, then $H$ is finitely generated since it is isomorphic to a subgroup of $T$. So assume that $K \neq 1$ and that $H \pi$ has finite index in $H_{1} \pi \cap H_{2} \pi$. By Proposition $4.1 \mathrm{~K}$ is finitely generated as a $\left(H_{1} \pi \cap H_{2} \pi\right)$-module. Since $H \pi$ has finite index in $H_{1} \pi \cap H_{2} \pi, K$ is also finitely generated as a $H \pi$-module.

This leads to the following decidability result:

Corollary 4.14 (=Corollary C). Let $W$ be the wreath product of two finitely generated free abelian groups. Let $H_{1}$ and $H_{2}$ be finitely generated subgroups of $W$. There is a uniform algorithm to determine whether or not $H_{1} \cap H_{2}$ is finitely generated, and, if so, whether or not $\mathrm{H}_{1} \cap \mathrm{H}_{2}$ is trivial.

Proof. We can compute finite hybrid presentations for $H_{1}$ and $H_{2}$, and from these, by Propositions 4.7 and 4.12, we can compute a finite hybrid presentation for $H_{1} \cap H_{2}$. From this finite hybrid presentation it is obvious whether $H_{1} \cap H_{2}$ are trivial since this is the case if and only if $H_{1} \cap H_{2} \cap B$ and $\left(H_{1} \cap H_{2}\right) \pi$ are both trivial. If $H_{1} \cap H_{2} \cap B$ is trivial, then $H_{1} \cap H_{2}$ is finitely generated. If $H_{1} \cap H_{2} \cap B$ is not trivial, then $H_{1} \cap H_{2}$ is finitely generated if and only if $\left(H_{1} \cap H_{2}\right) \pi$ has finite index in $H_{1} \pi \cap H_{2} \pi$, and this is easy to decide using linear algebra.

\section{Howson's theorem and some examples of intersections of finitely generated metabelian groups}

We again recall Howson's theorem [13] from 1954: if $F$ is a free group, and $H_{1}$ and $\mathrm{H}_{2}$ are finitely generated subgroups of $\mathrm{F}$, then $\mathrm{H}_{1} \cap \mathrm{H}_{2}$ is finitely generated.

Now instead let $F$ be a finitely generated free metabelian group. It is not difficult to produce two finitely generated subgroups of $F$ whose intersection is not finitely generated (one such example is provided below), so the obvious analog of Howson's theorem does not hold in this context. It is natural to then ask: which subgroups of a free metabelian group are more closely analogous to finitely generated subgroups of a free group? All subgroups of free groups are free, suggesting that there is an analog of Howson's theorem for finitely generated free metabelian subgroups of $F$. In any group, the intersection of two finite index subgroups is finitely generated by virtue of being of finite index itself. Another possibility then is to restrict attention to those free metabelian subgroups which are of finite index modulo the derived group of the given supergroup. Here we give one example that illustrates that Howson's theorem does not carry over to free metabelian groups in either instance. 
We construct two finitely generated subgroups of the free metabelian group $F$ that are themselves free, whose projections in $F /[F, F]$ generate all of $F /[F, F]$, and whose intersection is not finitely generated.

We again remark that the free metabelian subgroups of a finitely generated free metabelian group are of restricted rank. Specifically, if $F_{n}$ is the free metabelian group of rank $n$, then $F_{n}$ can not be embedded in $F_{n-1}$, the free metabelian group of rank $n-1$. In fact, more generally it can be shown that the free solvable groups $F_{n}\left(S_{\ell}\right)$ of rank $n$ and derived length $\ell$ cannot be embedded in a finite direct power of $F_{n-1}\left(S_{\ell}\right)$ for any $n$ (see Corollary 25.73 in [17]).

Theorem 5.1. Let $F$ be the free metabelian group of rank 2. Then there exist finitely generated free metabelian subgroups $H_{1}$ and $H_{2}$ of $F$ such that $H_{1}[F, F]=$ $H_{2}[F, F]=F$, but $H_{1} \cap H_{2}$ is not finitely generated. Moreover, $H_{1} \cap H_{2}=$ $H_{1} \cap B=H_{2} \cap B$ and is a free cyclic $\mathbb{Z}(F /[F, F])$-module.

Proof. Let $W$ be the wreath product of two finitely generated free abelian groups $A$ and $T$, each of rank 2 with bases $a_{1}, a_{2}$ and $t_{1}, t_{2}$ respectively. We adopt our usual notation: $B$ is the direct product of $\left\{A^{t} \mid t \in T\right\}$ that is normal in $W$ and $\pi$ is the projection of $W$ onto $T=W / B$.

Let $F$ be the subgroup group of $W$ that is generated by $t_{1} a_{1}$ and $t_{2} a_{2} . F$ is free metabelian [16]. Let $a$ be the commutator of the generators for $F$, so $a=$ $a_{1}^{t_{2}-1} a_{2}^{1-t_{1}} . F \cap B$ equals $[F, F]$ and is generated as a $T$-module by $a$. Let $H_{1}=$ $\left\langle t_{1} a_{1} a^{t_{1}}, t_{2} a_{2} a^{t_{2}}\right\rangle$ and let $H_{2}=\left\langle t_{1} a_{1} a, t_{2} a_{2} a\right\rangle$. Clearly $H_{1}$ and $H_{2}$ are subgroups of $F$ and $H_{1} \pi=H_{2} \pi=W \pi=T$. Furthermore $H_{1}[F, F]=H_{2}[F, F]=F$.

We will first show that $H_{1} \cap B=H_{2} \cap B \neq 1$. Let $b$ be the commutator of the generators of $H_{1}$. Then $H_{1} \cap B$ is generated as a $T$-module by $b$ where

$$
\begin{aligned}
b & =\left[t_{1} a_{1} a^{t_{1}}, t_{2} a_{2} a^{t_{2}}\right] \\
& =\left(a_{1} a^{t_{1}}\right)^{t_{2}-1}\left(a_{2} a^{t_{2}}\right)^{1-t_{1}} \\
& =a^{1+t_{1}\left(t_{2}-1\right)+t_{2}\left(1-t_{1}\right)} \\
& =a^{1-t_{1}+t_{2}} .
\end{aligned}
$$

But $H_{2} \cap B$ is generated as a $T$-module by $b$ as well since

$$
\left[t_{1} a_{1} a, t_{2} a_{2} a\right]=\left(a_{1} a\right)^{t_{2}-1}\left(a_{2} a\right)^{1-t_{1}}=a^{1+\left(t_{2}-1\right)+\left(1-t_{1}\right)}=a^{1-t_{1}+t_{2}} .
$$

Thus $H_{1} \cap B=H_{2} \cap B \neq 1$ as claimed.

Since $H_{1} \pi=H_{2} \pi=T$ and $H_{1} \cap B=H_{2} \cap B$ is the free cyclic $\mathbb{Z} T$-module generated by $b=a^{1-t_{1}+t_{2}}$, it follows that $H_{1}$ and $H_{2}$ are free metabelian groups [16]. Note that the free cyclic module $H_{1} \cap B$ is not finitely generated as a group (it is free abelian of countably infinite rank).

Finally, we will show that $\left(H_{1} \cap H_{2}\right) \leq B$, and hence that $H_{1} \cap H_{2}=H_{1} \cap B$ which we have already calculated. We define $d_{1}, d_{2}$ as in Section 4.2, so

$$
d_{i}=\left(t_{i} a_{i} a\right)^{-1}\left(t_{i} a_{i} a^{t_{i}}\right)=\left(a_{i} a^{t_{i}}\right)\left(a_{i} a\right)^{-1}=a^{t_{i}-1} .
$$


Let $n_{1}$ and $n_{2}$ be integers such that $t_{1}^{n_{1}} t_{2}^{n_{2}} \in\left(H_{1} \cap H_{2}\right) \pi$. Then by Proposition 4.9 we know that $d_{1}^{\left(n_{1} \theta_{1}\right) t_{2}^{n_{2}}} d_{2}^{n_{2} \theta_{2}}=a^{\left(1-t_{1}+t_{2}\right) f}$ for some $f \in \mathbb{Z} T$. Therefore

$$
\begin{aligned}
\left(t_{1}-1\right)\left(n_{1} \theta_{1}\right) t_{2}^{n_{2}}+\left(t_{2}-1\right)\left(n_{2} \theta_{2}\right) & =\left(1-t_{1}+t_{2}\right) f, \\
\left(t_{1}^{n_{1}}-1\right) t_{2}^{n_{2}}+\left(t_{2}^{n_{2}}-1\right) & =\left(1-t_{1}+t_{2}\right) f,
\end{aligned}
$$

and hence we have

$$
t_{1}^{n_{1}} t_{2}^{n_{2}}-1=\left(1-t_{1}+t_{2}\right) f .
$$

Such an equation is only possible if $n_{1}=n_{2}=0$ (and $f=0$ ). To see this observe that $\mathbb{Z} T$ is naturally embedded in $\mathbb{Q} T$. The retraction from $\mathbb{Q} T$ to $\mathbb{Q}$ defined by $t_{1} \mapsto 3$ and $t_{2} \mapsto 2$ sends the right-hand side of the equation to 0 . But the left-hand side is sent to $3^{n_{1}} 2^{n_{2}}-1$ which is 0 only when $n_{1}=n_{2}=0$, as claimed. Thus $\left(H_{1} \cap H_{2}\right) \leq B$, completing the proof of the theorem.

If $H_{1}$ and $H_{2}$ are subgroups satisfying the hypotheses of Proposition 5.1, then is it always the case that $H_{1} \cap H_{2}$ is not finitely generated? Our final example shows that the answer to this question is "No".

Proposition 5.2. Let $F$ be the free metabelian group of rank 2. Then there exist finitely generated free metabelian subgroups $H_{1}$ and $H_{2}$ of $F$ such that $H_{1}[F, F]=$ $H_{2}[F, F]=F, H_{1} \not \leq H_{2}, H_{2} \not \leq H_{1}$, and $H_{1} \cap H_{2}$ is finitely generated.

Proof. Let $W$ be the wreath product of two finitely generated free abelian groups $A$ and $T$, each of rank 2 with bases $a_{1}, a_{2}$ and $t_{1}, t_{2}$ respectively. We adopt our usual notation: $B$ is the direct product of $\left\{A^{t} \mid t \in T\right\}$ that is normal in $W ; \pi$ is the projection of $W$ onto $W / B ; \mathbb{Z} T$ is the group ring of $T$.

Let $F$ be the subgroup group of $W$ that is generated by $t_{1} a_{1}$ and $t_{2} a_{2} . F$ is free metabelian by [16]. Let $a$ be the commutator of the generators for $F$, so $a=$ $a_{1}^{t_{2}-1} a_{2}^{1-t_{1}}$. Note that $F \cap B$ equals $[F, F]$ and is generated as a $\mathbb{Z} T$-module by $a$.

Let $H_{1}=\left\langle t_{1} a_{1} a^{t_{1}}, t_{2} a_{2} a^{t_{1}}\right\rangle$ and let $H_{2}=\left\langle t_{1} a_{1} a, t_{2} a_{2} a\right\rangle$. It is clear that $H_{1} \pi=$ $H_{2} \pi=W \pi=T$ and also that $H_{1}[F, F]=H_{2}[F, F]=F$. We will see below that $H_{1} \cap B$ and $H_{2} \cap B$ are nontrivial, from which it follows [16] that $H_{1}$ and $H_{2}$ are free metabelian with bases the given generators. It remains to prove that $H_{1} \cap H_{2}$ is finitely generated and that $H_{1} \not \leq H_{2}$ and $H_{2} \not \leq H_{1}$.

We will begin by showing that $H_{1} \cap H_{2}$ is finitely generated. By Proposition 4.7 we know $H_{1} \cap H_{2} \cap B$ is finitely generated as a $T$-module. So it suffices to show that $\left(H_{1} \cap H_{2}\right) \pi=T$. Then by Proposition 4.9 it suffices to show that $t_{1} \mu=d_{1}$ and $t_{2} \mu=d_{2}$ both belong to $\left(H_{1} \cap B\right)\left(H_{2} \cap B\right)$. In the present example $d_{1}=$ $a_{1} a^{t_{1}}\left(a_{1} a\right)^{-1}$ and $d_{2}=a_{2} a^{t_{1}}\left(a_{2} a\right)^{-1}$, and so $d_{1}=d_{2}=a^{t_{1}-1}$. 
Now $H_{1} \cap B$ is generated as a $T$-module by

$$
\begin{aligned}
{\left[t_{1} a_{1} a^{t_{1}}, t_{2} a_{2} a^{t_{1}}\right] } & =a^{-t_{1}} a_{1}^{-1} t_{1}^{-1} a^{-t_{1}} a_{2}^{-1} t_{2}^{-1} t_{1} a_{1} a^{t_{1}} t_{2} a_{2} a^{t_{1}} \\
& =a_{1}^{t_{2}-1} a_{2}^{1-t_{1}} a^{-t_{1}^{2}+t_{1} t_{2}} \\
& =a^{1-t_{1}^{2}+t_{1} t_{2}} .
\end{aligned}
$$

Therefore $H_{1} \cap B$ consists of all elements $B$ of the form $a^{f}$ where $f$ is in the ideal $\mathcal{I}$ of $\mathbb{Z} T$ generated by $1-t_{1}^{2}+t_{1} t_{2}$. A similar calculation shows that $\left[t_{1} a_{1} a, a, t_{2} a_{2} a\right]=$ $a^{1-t_{1}+t_{2}}$. Therefore $H_{2} \cap B$ is generated by $a^{1-t_{1}+t_{2}}$, and hence $H_{2} \cap B$ consists of all elements $B$ of the form $a^{f}$ where $f$ is in the ideal $\mathcal{g}$ of $\mathbb{Z} T$ generated by $1-t_{1}+t_{2}$. Hence $\left(H_{1} \cap B\right)\left(H_{2} \cap B\right)$ is the set of all elements of $B$ of the form $a^{f}$ where $f$ is in the ideal $\mathcal{K}$ in $\mathbb{Z} T$ generated by $1-t_{1}^{2}+t_{1} t_{2}$ and $1-t_{1}+t_{2}$. Since $d_{1}=d_{2}=a^{t_{1}-1}$, it remains to show that $t_{1}-1$ is in $\mathcal{K}$. But this is clear since

$$
t_{1}-1=t_{1}\left(1-t_{1}+t_{2}\right)-\left(1-t_{1}^{2}+t_{1} t_{2}\right) .
$$

This completes the proof that $H_{1} \cap H_{2}$ is finitely generated.

Next we show that $H_{1} \not \leq H_{2}$ by showing that $1-t_{1}^{2}+t_{1} t_{2} \notin \mathcal{A}=\left(1-t_{1}+t_{2}\right)$.

$$
\begin{aligned}
1-t_{1}^{2}+t_{1} t_{2} \in \mathcal{J} & \Longleftrightarrow t_{1}^{-1}-t_{1}+t_{2} \in \mathcal{J} \\
& \Longleftrightarrow t_{1}^{-1}-1 \in \mathcal{A} \\
& \Longleftrightarrow 1-t_{1} \in \mathcal{A} \\
& \Longleftrightarrow t_{2} \in \mathcal{A} \\
& \Longleftrightarrow \mathcal{A}=\mathbb{Z} T,
\end{aligned}
$$

but this is the case if and only if $1-t_{1}+t_{2}$ is invertible in $\mathbb{Z} T$. But by [12], the invertible elements of $\mathbb{Z} T$ are all of the form $\pm t$ for some $t \in T$.

Next we show that $H_{2} \not \leq H_{1}$ by showing that $1-t_{1}+t_{2} \notin \mathcal{I}=\left(1-t_{1}^{2}+t_{1} t_{2}\right)$.

$$
1-t_{1}+t_{2} \in \mathcal{I} \Longleftrightarrow t_{1}-t_{1}^{2}+t_{1} t_{2} \in \mathcal{I} \Longleftrightarrow t_{1}-1 \in \mathcal{I} .
$$

Therefore, if $1-t_{1}+t_{2} \in \mathcal{I}$ then $t_{2} \in \mathcal{I}$, so $\mathcal{I}=\mathbb{Z} T$. Since $1-t_{1}+t_{2}$ is not invertible in $\mathbb{Z} T$, this is impossible. This completes the proof of the proposition.

A final example: Finally we construct an example which is of interest from several perspectives. Let $H=\langle a\rangle \mathrm{wr}\langle s\rangle$ be the wreath product of the cyclic group generated by $a$ with the cyclic group generated by $s$. If we introduce the abbreviation $a_{i}=a^{s^{i}}$ then $H$ can also be viewed as the split extension of the free abelian group $B$ with basis $\left\{a_{i}(i \in \mathbb{Z})\right\}$ by the infinite cycle on $s$ which acts by $a_{i} \mapsto a_{i+1}$. Also $H$ has a presentation as $H=\left\langle a, s \mid\left[a, a^{s^{i}}\right]=1, i \in \mathbb{Z}\right\rangle$ and is finitely generated but not finitely presented. 
Now we define $G$ to be the ascending HNN extension of $H$ by the stable letter $t$ which acts by $a \mapsto a^{2}$ and $s \mapsto s$. Thus

$$
\begin{aligned}
G & =\left\langle H, t \mid t^{-1} s t=s, t^{-1} a t=a^{2}\right\rangle \\
& =\left\langle a, s, t \mid\left[a, a^{s^{i}}\right]=1, t^{-1} s t=s, t^{-1} a t=a^{2}\right\rangle .
\end{aligned}
$$

Then $G$ is also finitely generated but not finitely presented. Notice that the subgroup generated by $\{a, t\}$ has the familiar presentation $B S(1,2)=\left\langle a, t \mid t^{-1} a t=a^{2}\right\rangle$ and has derived group isomorphic to the additive group of $\mathbb{Z}\left[\frac{1}{2}\right]$. It follows that the the derived group $[G, G]$ is the normal closure of the element $a$ and is isomorphic to the direct sum of countably many copies of $\mathbb{Z}\left[\frac{1}{2}\right]$ which are conjugate by the action of $s$.

We next consider the subgroup $K$ of $G$ generated by the two elements $\{a, s t\}$. If we abbreviate $u=s t$ then we calculate $u^{-k} a^{m} u^{k}=t^{-k} s^{-k} a^{m} s^{k} t^{k}=t^{-k} a_{k}^{m} t^{k}$. In case $k \geq 0$ this becomes $u^{-k} a^{m} u^{k}=a_{k}^{m 2^{k}}$. But if $k<0$ the $t^{k}$ or equivalently $u^{k}$ can only be pinched when $m=n 2^{|k|}$ for some $n$, and then $u^{-k} a^{n 2^{|k|}} u^{k}=a_{k}^{n}$. Consequently the elements of $K$ which are equal to $u$-free words lie in the subgroup of $B$ generated by the elements $\left\{\ldots, a_{-2}, a_{-1}, a_{0}, a_{1}^{2}, a_{2}^{4}, a_{3}^{8}, \ldots\right\}$.

Now observe the words of $H$ which represent elements of $K$ must have exponent sum 0 on $s$ and hence lie in $B$. It follows that $H \cap K$ is exactly the subgroup of $B$ which we have just described, that is,

$$
H \cap K=\left\langle\ldots, a_{-2}, a_{-1}, a_{0}, a_{1}^{2}, a_{2}^{4}, a_{3}^{8}, \ldots\right\rangle .
$$

Of course $G /[G, G]$ is the free abelian group on $\{s, t\} . H \cap K$ is closed under the action of positive powers of $t$ and of $u$ but not negative powers. It is also closed under negative powers of $s$ but not positive powers. More generally $H \cap K$ is not a submodule of $[G, G]$ for any non-trivial subgroup of $G /[G, G]$. So $H \cap K$ is the intersection of two finitely generated groups but is not finitely hybrid-presentable as a subgroup. Consequently not all of our results carry over to finitely generated metabelian groups in general. Notice however that $H \cap K$ is finitely generated by $\left\{a_{0}\right\}$ as a module over the polynomial ring $\mathbb{Z}\left[u, s^{-1}\right]$ with monoid generators $\left\{u, s^{-1}\right\}$. This suggests the notion of hybrid-presentable may need to be expanded to include such modules.

Since this group is generated by the subgroups $H$ and $K$, it follows that the subgroup $[H, K]$ is normal in $G$. Now $[a, s]=a_{0}^{-1} a_{1}$ and $[a, u]=a_{0}^{-1} a_{1}^{2}$, both lie in $[H, K]$ and so $a_{1}=a^{s} \in[H, K]$. Since $[H, K]$ is normal, it follows that $[H, K]=[G, G]$. One can also check that $[H, K] /(H \cap K)$ is isomorphic to the direct sum of countably many copies of the group of $2^{n}$-th roots of unity. 


\section{References}

[1] G. Baumslag, Some theorems on the free groups of certain product varieties. J. Combin. Theory 2 (1967), 77-99. Zbl 0237.20021 MR 0206077

[2] G. Baumslag, Subgroups of finitely presented metabelian groups. J. Austral. Math. Soc. 16 (1973), 98-110. Zbl 0287.20027 MR 0332999

[3] G. Baumslag, F. B. Cannonito, and C. F. Miller, III, Computable algebra and group embeddings. J. Algebra 69 (1981), 186-212. Zbl 0497.20023 MR 0613868

[4] G. Baumslag, F. B. Cannonito, and D. J. S. Robinson, The algorithmic theory of finitely generated metabelian groups. Trans. Amer. Math. Soc. 344 (1994), 629-648.

Zbl 0821.20019 MR 1202419

[5] G. Baumslag, D. Gildenhuys, and R. Strebel, Algorithmically insoluble problems about finitely presented solvable groups, Lie and associative algebras. I. J. Pure Appl. Algebra 39 (1986), 53-94. Zbl 0577.20021 MR 816890

[6] G. Baumslag, U. Stammbach, and R. Strebel, The free metabelian group of rank two contains continuously many nonisomorphic subgroups. Proc. Amer. Math. Soc. 104 (1988), 702. Zbl 0691.20027 MR 941322

[7] W. W. Boone, Certain simple, unsolvable problems of group theory. V, VI. Nederl. Akad. Wetensch. Proc. Ser. A. 60 = Indag. Math. 19 (1957), 22-27, 227-232. Zbl 0067.25706 MR 0098776

[8] J. R. J. Groves and C. F. Miller, III, Recognizing free metabelian groups. Illinois J. Math. 30 (1986), 246-254. Zbl 0598.20040 MR 840123

[9] P. Hall, Finiteness conditions for soluble groups. Proc. London Math. Soc. (3) 4 (1954), 419-436. Zbl 0056.25603 MR 0072873

[10] P. Hall, On the finiteness of certain soluble groups. Proc. London Math. Soc. (3) 9 (1959), 595-622. Zbl 0091.02501 MR 0110750

[11] O. G. Kharlampovič, A finitely presented solvable group with unsolvable word problem. Izv. Akad. Nauk SSSR Ser. Mat. 45 (1981), 852-873; English transl. Math. USSR-Izv. 19 (1982), 151-169. Zbl 0494.20021 MR 0631441

[12] G. Higman, The units of group-rings. Proc. London Math. Soc. (2) 46 (1940), 231-248. JFM 66.0104.04 Zbl 0025.24302 MR 0002137

[13] A. G. Howson, On the intersection of finitely generated free groups. J. London Math. Soc. 29 (1954), 428-434. Zbl 0056.02106 MR 0065557

[14] A. S. Kirkinskiı̌, Intersections of finitely generated subgroups in metabelian groups. Algebra i Logika 20 (1981), 37-54; English transl. Algebra and Logic 20 (1981), 24-36. Zbl 0476.20022 MR 0635649

[15] J. C. Lennox and D. J. S. Robinson, The theory of infinite soluble groups. Oxford Math. Monogr., Oxford University Press, Oxford 2004. Zbl 1059.20001 MR 2093872

[16] W. Magnus, On a theorem of Marshall Hall. Ann. of Math. (2) 40 (1939), 764-768. JFM 65.0068.01 Zbl 0022.31403 MR 0000262

[17] H. Neumann, Varieties of groups. Ergeb. Math. Grenzgeb. (2) 37, Springer-Verlag, Berlin 1967. Zbl 0251.20001 MR 0215899 
[18] G. A. Noskov, Conjugacy problem in metabelian groups. Mat. Zametki 31 (1982), 495507; English transl. Math. Notes 31 (1982) , 252-258. Zbl 0492.20018 MR 657712

[19] P. S. Novikov, On the algorithmic insolvability of the word problem in group theory. Trudy Mat. Inst. im. Steklov. 44, Izdat. Akad. Nauk SSSR, Moscow 1955; English transl. Amer. Math. Soc. Transl. (2) 9 (1958), 1-122. Zbl 0068.01301 MR 0075197

[20] V. N. Remeslennikov, On finitely presented groups. In Proc. fourth all-union symposium on group theory, Novosibirsk. Gos. Univ., Novosibirsk 1973, 164-169.

[21] N. S. Romanovskii, The occurrence problem for extensions of Abelian groups by nilpotent groups. Sibirsk. Mat. Zh. 21 (1980), 170-174; English transl. Siberian Math. J. 21 (1980), 273-276. Zbl 0469.20019 MR 569186

Received October 12, 2009; revised March 1, 2010

G. Baumslag, Department of Mathematics and Computer Science, City College of New York, Convent Ave. and 138th Street, New York, NY 10031, U.S.A.

E-mail: gilbert@groups.sci.ccny.cuny.edu

C. F. Miller III, Department of Mathematics and Statistics, University of Melbourne, Melbourne, Victoria 3010, Australia

E-mail: c.miller@ms.unimelb.edu.au

G. Ostheimer, Department of Computer Science, 211 Adams Hall, Hofstra University, Hempstead NY 11549, U.S.A.

E-mail: cscgzo@hofstra.edu 